\title{
BMJ Open Functional MRI in the effect of transcranial magnetic stimulation therapy for patients with schizophrenia: a meta-analysis protocol
}

\author{
Siqian Zhong, Yiru Hu, Yu Fu, Liping Cao, Bin Zhang
}

To cite: Zhong S, Hu Y, Fu Y, et al. Functional MRI in the effect of transcranial magnetic stimulation therapy for patients with schizophrenia: a metaanalysis protocol. BMJ Open 2020;10:e038557. doi:10.1136/ bmjopen-2020-038557

- Prepublication history for this paper is available online. To view these files, please visit the journal online (http://dx.doi. org/10.1136/bmjopen-2020038557).

SZ and YH contributed equally.

Received 15 March 2020

Revised 03 November 2020 Accepted 12 November 2020

Deck for updates

(c) Author(s) (or their employer(s)) 2020. Re-use permitted under CC BY-NC. No commercial re-use. See rights and permissions. Published by BMJ.

PsyNI Lab, Affiliated Brain Hospital of Guangzhou Medical University, Guangzhou, China

Correspondence to

Dr Bin Zhang;

zhang.bin845@foxmail.com

\section{ABSTRACT}

Introduction Schizophrenia is a psychiatric illness associated with brain function alterations and varying degree of treatment resistance, often leading to severe social malfunctioning. In recent decades, numerous studies have been investigating the therapeutic potential of transcranial magnetic stimulation (TMS) as a noninvasive therapy for schizophrenia. However, its clinical efficacy remains controversial, as a number of clinical trials indicated moderate therapeutic effect while others failed to reproduce the positive result. Moreover, the neurobiological mechanism of action remains unclear, possibly constricting the application of TMS in clinical practice. The present protocol of meta-analysis aims to investigate the TMS-related functional neuroimaging (ie, functional MRI) features and alterations in subjects with schizophrenia, and to discuss the potential of functional MRI in TMS researches.

Methods and analysis The study selection process will follow the Preferred Reporting Items for Meta-Analyses guideline and quality assessment will be conducted with a customised checklist. We plan to search in the following databases: PubMed, Embase, OVID, China National Knowledge Infrastructure and Wanfang Data, from their respective dates of inception to 1 May 2020, with language restricted to English and Chinese. Studies focusing on the brain functional alterations in patients with schizophrenia treated by TMS will be retrieved.

Ethics and dissemination This work does not require ethics approval as it will be based on published studies. This systematic review will be publicly disseminated in peer-reviewed journals.

PROSPERO registration number CRD42020166288.

\section{INTRODUCTION}

Known for its prominent psychotic symptoms such as hallucination and delusion, schizophrenia (SZ), with a lifetime prevalence of $0.30 \%-0.66 \%$ in the general population, ${ }^{1-3}$ is a severe psychiatric illness that tends to be chronic and recurrent, leading to varying degrees of cognitive impairment and social disability.

Since a substantial proportion of patients are resistant to first-line antipsychotics,
Strengths and limitations of this study

- This is a novel meta-analysis focusing particularly on brain functional MRI in the transcranial magnetic stimulation (TMS) therapy for schizophrenia.

- This study will provide informative evaluation of the clinical efficacy of repetitive TMS and shed light on its neural substrate of action.

- The present protocol mainly focuses on the brain functional changes and the general symptomatic improvement induced by TMS, therefore fails to discuss the effect of different stimulation parameters.

- The limit of this study is connected with the crossstudy heterogeneity that affects the external validity.

We use public data that will be freely available after publication.

pharmacological interventions are sometimes insufficient in the treatment for SZ. ${ }^{4}$ As a noninvasive neurostimulation technique, transcranial magnetic stimulation (TMS) seems to be a promising add-on therapy that regulates brain function by activating or suppressing neural activity in an effective and safe manner, even though the evidences are controversial. A number of previous studies have explored the role of TMS in the treatment of cognitive deficit, ${ }^{5}$ auditory hallucinations (AH) and negative symptoms. ${ }^{6}$ For instance, 20 $\mathrm{Hz}$ repetitive TMS (rTMS) over left dorsolateral prefrontal cortex (DLPFC) was considered a safe and well-tolerated treatment for negative symptoms and cognitive deficit of SZ. ${ }^{7}$ However, a randomised, double-blind and sham-controlled trial suggested that therapeutic rTMS administered to the DLPFC in SZ did not result in evident cognitive enhancing effects. ${ }^{9}$ Data from several studies using functional MRI (fMRI) have supported that rTMS on language-perception area can alleviate $\mathrm{AH}$ without any impact on functional connectivity (FC) within the language network, ${ }^{10-12}$ while few other studies have found the FC alteration 
as the symptom relieved. ${ }^{13-16}$ In an update (2014-2018) of the evidence-based guidelines on the therapeutic use of rTMS, experts reviewed its curative effect on AH and negative symptoms; unfortunately, the strong heterogeneity between studies failed to support an extensive clinical application of such treatment. ${ }^{17}$

In recent studies of meta-analysis, researchers believe that the placebo effect, publication bias and the frequently mentioned cross-trial heterogeneity contributed to the uncertain efficacy of TMS therapy for SZ. ${ }^{5} 151618$ Therefore, in addition to focusing solely on the change of symptomatic features, further explorations on optimisation of stimulation parameters, as well as the neurobiological factors associated with treatment response, are of clinical necessity, so as to provide experiment basis for reliable and effective treatment strategies.

In fact, neuroimaging technique plays an important role in evaluating the therapeutic efficacy of TMS and exploring its neurobiological mechanism of action. A systematic review including 12 studies - 3 of which involving fMRI-discussed the major contributions of neuroimaging to TMS research in mainly two aspects: (1) for guiding the coil placement, and (2) for understanding the functional activation and connectivity in SZ. ${ }^{19}$ For example, according to some later studies, the treatment targets for $\mathrm{AH}$ are mostly identified by performing a language task during fMRI. ${ }^{140}$ In a 2012 study, the n-back task was performed twice on a group of patients during the pretreatment and post-treatment fMRI scan, in order to investigate treatment-related brain activation. ${ }^{21}$ Another brain connectivity and $\mathrm{AH}$ review indicated that FC alterations of the default-mode network may be related to hallucinations, and therefore rTMS - with its neuromodulatory effects on targeted cortical sites and their associated networks-is a promising treatment option for symptoms associated with altered connectivity in SZ. ${ }^{22}$ In addition, a multimodal fMRI-rTMS study demonstrated changes in cortical plasticity in human during executive cognition. ${ }^{23}$

Despite the extensive fMRI research we mentioned earlier, considerable variability of results exists between these studies, and as far as we are aware of, there has been no meta-analysis to date on studies investigating brain functional alteration induced by therapeutic TMS for SZ. Here we intend to describe the protocol for a metaanalysis aiming to summarise the TMS-related functional neuroimaging features in subjects with SZ, and consequently, to evaluate its clinical efficacy and explore the neural mechanism of action.

\section{OBJECTIVE}

This meta-analysis aims to integrate and assess the features of brain function in SZ after receiving TMS.

\section{METHODS}

This protocol follows the Preferred Reporting Items for Systematic Reviews and Meta-Analysis Protocols 2015 statement. $^{24}$

\section{Eligibility criteria}

Study types

Cohort studies and randomised controlled trials will be included only if the neuroimaging results are available. Case-control studies, case reports, narrative or systematic reviews, meta-analyses, letters and other secondhand studies will be excluded.

\section{Study design}

Both resting-state and task neuroimaging studies investigating functional activity or connectivity in patients with SZ treated with active or sham-TMS will be included. Longitudinal studies focusing on the management of SZ will also be considered, but only if the baseline neuroimaging data are available. Studies that (1) did not report whole-brain analyses, or (2) did not report coordinates in either Montreal Neurological Institute (MNI) or Talairach space, or (3) solely focusing on brain structure, will all be excluded.

\section{Participants}

Studies with subjects with SZ with or without a healthy control group (all without any known severe neurological condition or head trauma) are required. The minimum sample size will be 12 subjects per group, according to previous studies. ${ }^{25} 26$ Race, age and medication (ie, drugnaïve or medicated) will not be restricted.

\section{Interventions}

Active TMS or rTMS, and sham-TMS.

\section{Outcome measures}

Since we focus on therapeutic TMS-induced neuroimaging alteration in SZ, the main outcomes of the included studies are brain regions that indicate significant functional changes, including whole-brain functional activity or FC, and should be presented in MNI or Talairach coordinates.

The secondary result will be psychotic symptom improvement indexed by the score of Positive and Negative symptom Scale, ${ }^{27}$ the Auditory Hallucination Rating Scale or any other clinical assessment scales (eg, the Manchester Scale) reported in the included studies.

\section{Report characteristics}

Only published and peer-reviewed original studies in English or Chinese will be included. The date of publication should be prior to 1 May 2020, the anticipated completion date of this review.

\section{Searching strategy}

The following sources will be searched: PubMed, OVID, Embase, China National Knowledge Infrastructure (CNKI) and Wanfang Data, using Medical Subject Headings terms. The searching strategies of PubMed (English) and CNKI (Chinese) are presented in table 1 and will be applied to the other databases. 
Table 1 Searching strategy

\begin{tabular}{ll} 
PubMed searching strategy & CNKI searching strategy \\
\hline \#1 Schizophrenia (MeSH terms) & \#1精神分裂症 (主题词) \\
\#2 Schizophrenia (all fields) & \#2经频磁刺激 (主题词 ) \\
\#3 Schizophren* (all fields) & \#3重复经颅磁刺激 (主题词 ) \\
\#4 \#1 OR \#2 OR \#3 & \#4 \#2 OR \#3 \\
\#5 Transcranial Magnetic Stimulation (MeSH terms) & \#5神经影像学 \\
\#6 TMS (all fields) & \#6磁共振成像 (主题词 ) \\
\#7 \#5 OR \#6 & \#7功能磁共振成像 (主题词) \\
\#8 Functional Neuroimaging (MeSH terms) & \#8 fMRI (主题词 ) \\
\#9 Functional Neuroimaging (all fields) & \#9 \#5 OR \#6 OR \#7 OR \#8 \\
\#10 Functional Magnetic resonance* (MeSH terms) & \#10 Final search terms:\#1 AND \#4 AND \#9 \\
\#11 fMRI (all fields) & \\
\#12 \#8 OR \#9 OR \#10 OR \#11 & \\
\#13 Final search terms: \#4 AND \#7 AND \#12 & \\
\hline
\end{tabular}

CNKI, China National Knowledge Infrastructure; MeSH, Medical Subject Headings.

\section{Selection process}

EndNote V.X7 software (Thomson Reuters, New York, New York, USA) will be used to manage literature. After removing duplicates, studies identified by the literature search will be removed independently by SZ and YF, based on title and abstract. Then, the rest of the literature will be assessed by full-text screening for the final inclusion for this review. Any disagreement between the two reviewers will be reconsidered by a third reviewer, BZ. Reasons for study exclusion will be reported.

\section{Data collection}

The two independent reviewers (SZ and YF) will doubly extract data using a standard data extraction spreadsheet in Excel. Again, any inconsistency between reviewers will be reconsidered and the result determined by the third reviewer (BZ). The following items will be extracted from each record. (1) Publication information: title, first author, publishing time, unit, country or region, and funding support. (2) Details of methodology: participants, sample size, diagnostic criteria, demographic characteristics (including age, gender and so on), imaging modalities, scanner resolution, data analysis strategies, clinical assessments and clinical variables (eg, illness duration and severity, and so on). (3) Results: the significantly altered cerebral regions (defined by MNI/Talairach coordinates, cluster size and statistical threshold), the results of clinical assessments, and the correlations between imaging data and clinical data.

Any missing information or questions about the above data will be settled by contacting the authors. If no clarification is provided within 4 weeks, the study will be included in the final analysis with its missing information reported.

\section{Quality assessment}

So far, there has been no standard checklist for quality assessment of individual functional neuroimaging studies. We will adopt a checklist (see box 1) published in a previous meta-analysis. ${ }^{28} 29$

Two independent reviewers (SZ and YF) will examine for any potential study bias, such as the characteristics of

\section{Box 1 Quality assessment of individual studies}

\section{Category 1: sample characteristics (10)}

- Patients were evaluated with specific standardised diagnostic criteria (1).

- Important demographic data (age and gender) were reported with mean (or median) and SDs (or range) (2).

- Healthy control subjects were evaluated to exclude psychiatric and medical illnesses and demographic data were reported (1).

- Important clinical variables (eg, positive and negative symptom, medication status, and illness duration and severity) were reported with mean (or median) and SDs (or range) (4).

- Sample size per group $>10$ (2).

\section{Category 2: methodology and reporting (10)}

- Whole brain analysis was automated with no a priori regional selection (3).

- Magnet strength at least 1.5T (1).

- At least 5 min of resting state acquisition (1).

- Whole brain coverage of resting scans (1).

- The acquisition and preprocessing techniques were clearly described so that they could be reproduced (1).

- Coordinates reported in a standard space (1).

- Significant results are reported after correction for multiple testing using a standard statistical procedure (AlphaSim, FDR, FWE or permutation-based methods) (1).

- Conclusions were consistent with the results obtained and the limitations were discussed (1).

FDR, false discovery rate; FWE, family-wise error rate. 
the sample, the methods of randomisation and blinding, the completeness of outcome data and others, using the Cochrane Collaboration's tool. The assessment will be done at study level. Inconsistencies between SZ and YF will be settled by discussions with the third reviewer (BZ).

\section{Data synthesis}

All collected data will be put together in a table, including the total and average sample size, age range of subjects, mean duration of illnesses, as well as the clinical assessment results. We will perform a qualitative analysis to examine whether the different functional patterns exist in SZ after the TMS treatment compared with the baseline. Also, we will summarise these results by task-based or resting-state fMRI.

\section{Data analysis}

First, the coordinates in MNI and Talairach space extracted from the included studies will be converted to one another for the convenience of our analysis. An activation likelihood estimation (ALE) meta-analysis will then be performed to integrate consistent brain regions with significant functional alteration reported in different studies, using GingerALE V.3.0.2 (http://www. brainmap.org/). In the ALE algorithm, the peak coordinates extracted from studies represent their own clusters and were registered as centres in the 3D Gaussian probability distribution. The sizes of these clusters were estimated with experimental design of each study, including sample sizes, between-subject variations, normalising methods and others. Finally, an ALE map was calculated by merging all modelled activation maps obtained through voxel-wise aggregation of all clusters reported in each experiment. This ALE map was then compared against a randomly permuted null-distribution to test for statistical significance.

To assess the stability of the outcomes, the leave-one-out jackknife sensitivity analysis will be performed using GingerALE. Specifically, this is a procedure that iteratively recalculate the effect size by excluding a different study from the sample at a time and then repeating the analyses. If necessary, subgroup analysis will be performed based on different stimulation parameters of TMS (frequency, intensity and others) and courses of the disease (ie, firstepisode or chronic SZ).

Contributors BZ was responsible for this study. SZ, YH and BZ conceived and designed the study. BZ, SZ, YF and LC participated in drafting the protocol and preparing the manuscript. SZ, YH, YF, LC and BZ read and approved the final manuscript.

Funding This work is partly funded by National Key R\&D Program of China (2016YFC1306804).

Competing interests None declared.

Patient and public involvement Patients and/or the public were not involved in the design, or conduct, or reporting, or dissemination plans of this research.

Patient consent for publication Not required.

Provenance and peer review Not commissioned; externally peer reviewed.

Open access This is an open access article distributed in accordance with the Creative Commons Attribution Non Commercial (CC BY-NC 4.0) license, which permits others to distribute, remix, adapt, build upon this work non-commercially, and license their derivative works on different terms, provided the original work is properly cited, appropriate credit is given, any changes made indicated, and the use is non-commercial. See: http://creativecommons.org/licenses/by-nc/4.0/.

ORCID iD

Bin Zhang http://orcid.org/0000-0002-9280-8247

\section{REFERENCES}

1 Owen MJ, Sawa A, Mortensen PB. Schizophrenia. Lancet 2016;388:86-97.

2 American Psychiatric Association. Diagnostic and statistical manual of mental disorders(DSM-5). Washington, DC: American PsychiatricAssociation, 2013. https://doi.org/10.1176/appi.books

3 McGrath J, Saha S, Chant D, et al. Schizophrenia: a Concise overview of incidence, prevalence, and mortality. Epidemiol Rev 2008;30:67-76.

4 Lehman AF, Lieberman JA, Dixon LB, et al. Practice guideline for the treatment of patients with schizophrenia, second edition. Am J Psychiatry 2004;161:1-56.

5 Jiang Y, Guo Z, Xing G, et al. Effects of high-frequency transcranial magnetic stimulation for cognitive deficit in schizophrenia: a metaanalysis. Front Psychiatry 2019;10:135.

$6 \mathrm{He} \mathrm{H}$, Lu J, Yang L, et al. Repetitive transcranial magnetic stimulation for treating the symptoms of schizophrenia: a PRISMA compliant meta-analysis. Clin Neurophysiol 2017;128:716-24.

7 Guan HY, Zhao JM, Wang KQ, et al. High-Frequency neuronavigated rTMS effect on clinical symptoms and cognitive dysfunction: a pilot double-blind, randomized controlled study in veterans with schizophrenia. Trans/ Psychiatry 2020;10:79.

8 Zhuo K, Tang Y, Song Z, et al. Repetitive transcranial magnetic stimulation as an adjunctive treatment for negative symptoms and cognitive impairment in patients with schizophrenia: a randomized, double-blind, sham-controlled trial. Neuropsychiatr Dis Treat 2019;15:1141-50.

9 Martin DM, McClintock SM, Forster J, et al. Does therapeutic repetitive transcranial magnetic stimulation cause cognitive enhancing effects in patients with neuropsychiatric conditions? A systematic review and meta-analysis of randomised controlled trials. Neuropsychol Rev 2016;26:295-309.

10 Giesel FL, Mehndiratta A, Hempel A, et al. Improvement of auditory hallucinations and reduction of primary auditory area's activation following TMS. Eur J Radiol 2012;81:1273-5.

11 Briend F, Leroux E, Delcroix N, et al. Impact of rTMS on functional connectivity within the language network in schizophrenia patients with auditory hallucinations. Schizophr Res 2017;189:142-5.

12 Gromann PM, Tracy DK, Giampietro V, et al. Examining frontotemporal connectivity and rTMS in healthy controls: implications for auditory hallucinations in schizophrenia. Neuropsychology 2012;26:127-32.

13 Slotema CW, Blom JD, de Weijer AD, et al. Can low-frequency repetitive transcranial magnetic stimulation really relieve medicationresistant auditory verbal hallucinations? negative results from a large randomized controlled trial. Biol Psychiatry 2011;69:450-6.

14 Paillère-Martinot M-L, Galinowski A, Plaze M, et al. Active and placebo transcranial magnetic stimulation effects on external and internal auditory hallucinations of schizophrenia. Acta Psychiatr Scand 2017;135:228-38.

15 Dollfus S, Lecardeur L, Morello R, et al. Placebo response in repetitive transcranial magnetic stimulation trials of treatment of auditory hallucinations in schizophrenia: a meta-analysis. Schizophr Bull 2016;42:301-8.

16 Wang J, Zhou Y, Gan H, et al. Efficacy towards negative symptoms and safety of repetitive transcranial magnetic stimulation treatment for patients with schizophrenia: a systematic review. Shanghai Arch Psychiatry 2017;29:61-76.

17 Lefaucheur J-P, André-Obadia N, Antal A, et al. Evidence-Based guidelines on the therapeutic use of repetitive transcranial magnetic stimulation (rTMS). Clin Neurophysiol 2014;125:2150-206.

18 Kennedy NI, Lee WH, Frangou S. Efficacy of non-invasive brain stimulation on the symptom dimensions of schizophrenia: a meta-analysis of randomized controlled trials. Eur Psychiatry 2018;49:69-77.

19 Du Z-de, Wang R, Prakash R, et al. Transcranial magnetic stimulation in schizophrenia: the contribution of neuroimaging. Curr Top Med Chem 2012;12:2452-7.

20 Bais L, Liemburg E, Vercammen A, et al. Effects of low frequency rTMS treatment on brain networks for inner speech in patients 
with schizophrenia and auditory verbal hallucinations. Prog Neuropsychopharmacol Biol Psychiatry 2017;78:105-13.

21 Guse B, Falkai P, Gruber O, et al. The effect of long-term high frequency repetitive transcranial magnetic stimulation on working memory in schizophrenia and healthy controls--a randomized placebo-controlled, double-blind fMRI study. Behav Brain Res 2013;237:300-7.

22 Thomas F, Moulier V, Valéro-Cabré A, et al. Brain connectivity and auditory hallucinations: in search of novel noninvasive brain stimulation therapeutic approaches for schizophrenia. Rev Neurol 2016;172:653-79.

23 Esslinger C, Schüler N, Sauer C, et al. Induction and quantification of prefrontal cortical network plasticity using $5 \mathrm{~Hz}$ rTMS and fMRI. Hum Brain Mapp 2014;35:140-51.

24 Moher D, Shamseer L, Clarke M, et al. Preferred reporting items for systematic review and meta-analysis protocols (PRISMA-P) 2015 statement. Syst Rev 2015;4:1.
25 Desmond JE, Glover GH. Estimating sample size in functional MRI (fMRI) neuroimaging studies: statistical power analyses. J Neurosci Methods 2002;118:115-28.

26 Yin T, Li Z, Xiong J, et al. Neuroimaging biomarkers of psychogenic erectile dysfunction: protocol for a systematic review. BMJ Open 2019;9:e030061.

27 Kay SR, Fiszbein A, Opler LA, et al. The positive and negative syndrome scale (PANSS) for schizophrenia. Schizophr Bull 1987;13:261-76.

28 Gong J, Wang J, Luo X, et al. Abnormalities of intrinsic regional brain activity in first-episode and chronic schizophrenia: a metaanalysis of resting-state functional MRI. J Psychiatry Neurosci 2020;45:55-68.

29 Li S, Hu N, Zhang W, et al. Dysconnectivity of multiple brain networks in schizophrenia: a meta-analysis of resting-state functional connectivity. Front Psychiatry 2019;10:482. 